\title{
Monitoring radiofrequency ablation of biological tissue using broadband time-resolved diffuse optical spectroscopy
}

Lanka, Pranav, Francis, K., Kruit, Hindrik, Konugolu Venkata Sekar, Sanathana, Farina, Andrea, et al.

Pranav Lanka, K. J. Francis, Hindrik Kruit, Sanathana Konugolu Venkata Sekar, Andrea Farina, Rinaldo Cubeddu, Srirang Manohar, Antonio Pifferi, "Monitoring radiofrequency ablation of biological tissue using broadband timeresolved diffuse optical spectroscopy," Proc. SPIE 11074, Diffuse Optical Spectroscopy and Imaging VII, 110742M (11 July 2019); doi: $10.1117 / 12.2526964$ 


\title{
Monitoring radiofrequency ablation of biological tissue using broadband time-resolved diffuse optical spectroscopy
}

\author{
Pranav Lanka*a , K. J. Francis ${ }^{\mathrm{b}}$, Hindrik Kruit ${ }^{\mathrm{b}}$, Sanathana Konugolu Venkata Sekar ${ }^{\mathrm{a}}$, \\ Andrea Farina $^{c}$, Rinaldo Cubeddu ${ }^{\mathrm{a}}$, Srirang Manohar ${ }^{\mathrm{b}}$ Antonio Pifferi $^{\mathrm{a}, \mathrm{c}}$ \\ ${ }^{a}$ Politecnico di Milano, Dipartimento di Fisica, Piazza Leonardo da Vinci 32, 20133 Milano (Italy); \\ ${ }^{\mathrm{b}}$ Biomedical Photonic Imaging Group, Technical Medical Centre, University of Twente, \\ Enschede,Netherlands; 'Consiglio Nazionale delle Ricerche, Istituto di Fotonica e Nanotecnologie, \\ Piazza Leonardo da Vinci 32, 20133 Milano (Italy)
}

\begin{abstract}
Radiofrequency ablation (RFA) is minimally invasive thermotherapy, where a heating source is used to target and kill malignant cells in a tissue. While RFA has tremendous potential in the field of oncology, there is also a need for reliable real-time monitoring of this procedure to avoid over or under treatment. In this work, we investigate the use of timeresolved diffuse optical spectroscopy (DOS) to continuously track the change in optical properties during RFA to monitor the process of ablation. The time evolution of the spectra of the optical properties of the tissue undergoing treatment gives deep insights into the structural and constitutional changes occurring during the RFA treatment.
\end{abstract}

Keywords: Diffuse optical spectroscopy, absorption, liver, radio frequency ablation, light scattering, non-invasive, monitoring

\section{INTRODUCTION}

Thermotherapy has shown considerable promise in cancer treatment. Based on the nature of the heating source different modalities have been developed such as Radio Frequency Ablation (RFA), Micro Wave Ablation, High Intensity Focused Ultrasound, Laser Ablation, and Cryoablation. RFA is a minimally invasive treatment where a needle probe is inserted percutaneously into the tumor. An alternating current is then applied through the needle probe inducing ionic agitation and resultant heating of the tumor site. Controlled heating for a specific duration can result in coagulative necrosis of malignant tissue. This technique has been successful in treating many diseases and is widely used to treat cancers in the liver, lungs, breast, and bone ${ }^{1}$. However, one major drawback of the RFA is the lack of tools for real-time monitoring of the treatment. Real-time ablation monitoring is necessary to improve the efficiency of the treatment and avoid unwanted risks ${ }^{2}$. While the temperature of the needle probe and tissue impedance monitoring could be of some help in this regard, there exists a need for more accurate ablation monitoring to improve treatment efficiency and to reduce the tumor recurrence ${ }^{2,3}$.

Time-resolved Diffuse optical spectroscopy (TDOS) is a valuable technique that has been extensively used in-vivo for the non-invasive monitoring and diagnosis of pathologies in organs like the breast and forehead ${ }^{4,5}$. In this work, we explored the use of broadband TDOS to monitor radiofrequency ablation in biological tissue. In particular, we performed transmittance measurements on the ex-vivo bovine liver and turkey breast tissue undergoing RFA, to see the effect it has on the retrieved absorption and scattering coefficient spectra.

\section{MATERIALS AND METHODS}

\subsection{Instrumentation:}

An in-house built broadband time domain spectrometer was used for this study. A detailed description of the instrument is provided elsewhere ${ }^{6}$. A supercontinuum fiber laser (SuperK, NKT Photonics, Denmark), with a spectral range of 450$1750 \mathrm{~nm}$ generating picosecond pulses (40 MHz repetition rate), was used as the source. Spectral tunability is achieved

Diffuse Optical Spectroscopy and Imaging VII, edited by Hamid Dehghani, Heidrun Wabnitz, Proc. of SPIE-OSA Vol. 11074, 110742M · C 2019 SPIE-OSA · CCC code: 1605-7422/19/\$21 · doi: 10.1117/12.2526964 
by dispersing the source with a Pellin-Broca prism and coupling the selected wavelength into the injection fiber. A Silicon Photomultiplier (SiPM) detector with light harvesting capabilities over the wavelength range of $600-1100 \mathrm{~nm}$ was used as the detector in conjunction with a time-correlated single photon counting board (SPC-130, Becker \& Hickl, Germany). The injection and collection fibers were bare plastic-glass multimode fibers with $1 \mathrm{~mm}$ core diameter. An RF ablation system (Angiodynamics 1500x) with multiple electrode probe (StarBurst XL) with temperature feedback system was used for the RFA treatment of the samples.

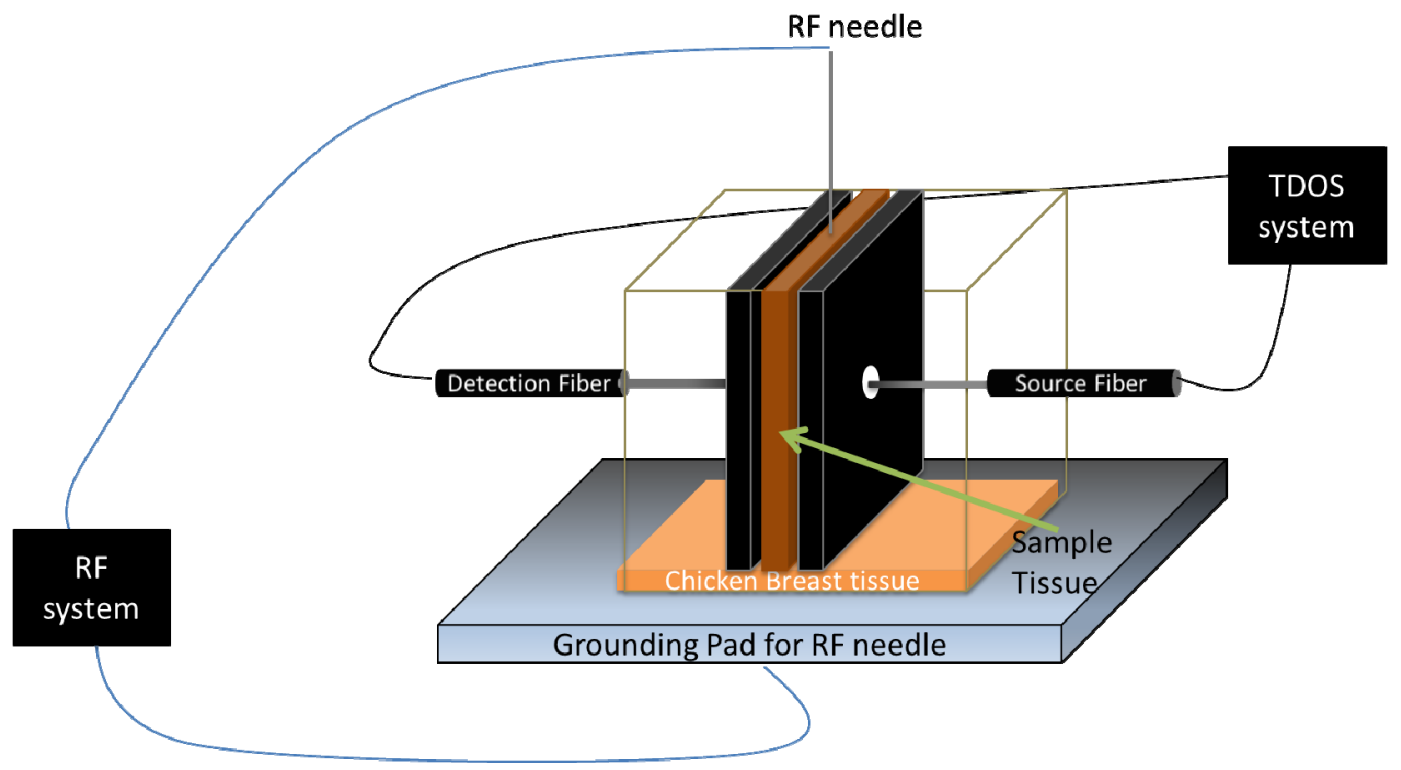

Figure 1. Schematic of the setup

\subsection{Sample Preparation and Measurement:}

Ex-vivo bovine liver and turkey breast tissue was used for this study. Multiple samples were prepared from the same organ as rectangular slabs with a thickness of $1,1.5$ and $2 \mathrm{~cm}$. Figure 1 shows a schematic of the setup. The samples were sandwiched between two black PVC plates that were locked using nylon screws on all four corners to keep the thickness intact and avoid any movement. Care was taken not to squeeze the sample, which could change its structural and optical properties. The PVC plates had two small openings of $1 \mathrm{~mm}$ diameter at their centers to house the injection and collection fibers on either side of the sample. This setup was used to perform the TDOS measurements in transmittance geometry. A glass tank with aluminum base was used for RFA procedure. A layer of chicken breast tissue was used to establish uniform contact between the sample and the aluminum grounding plate of the glass tank. The RF needle was inserted into the sample in a direction perpendicular to the source and detector fibers. The multiple electrode extensions of the needle were then deployed to uniformly ablate the volume of the tissue that is directly in the field of view of the TDOS setup. Depending on the thickness of the tissue sample the tines were radially deployed to fill the entire thickness, yet avoiding contact with the PVC plates. All measurements were performed in transmittance geometry. The broadband spectra $(600-1100 \mathrm{~nm})$ obtained during the thermal treatment were acquired in intervals of $1 \mathrm{~min}$ while continuous monitoring was performed at a single wavelength $(1064 \mathrm{~nm})$. Here we present results only from the spectral measurements.

\section{RESULTS AND CONCLUSION}

Figure 2 shows the time evolution of the spectra of the optical properties of turkey breast tissue during RF treatment at $70^{\circ} \mathrm{C}$. The absorption spectra (a) shows a decreasing trend which appears to reach a minimum after a few minutes of heat treatment. Specifically, we observe a redshift of the hemoglobin peak at $760 \mathrm{~nm}$ and a blue shift of the water peak at 
$970 \mathrm{~nm}$ as a consequence of the ablation. One other interesting feature is the emergence of a new peak at $840 \mathrm{~nm}$ which becomes more evident with increasing treatment time. We observe a gradual increase both in the amplitude and the slope of the scattering spectrum (b) with treatment could be an important indicator of the structural changes the occur in the tissue over the period of the ablation.

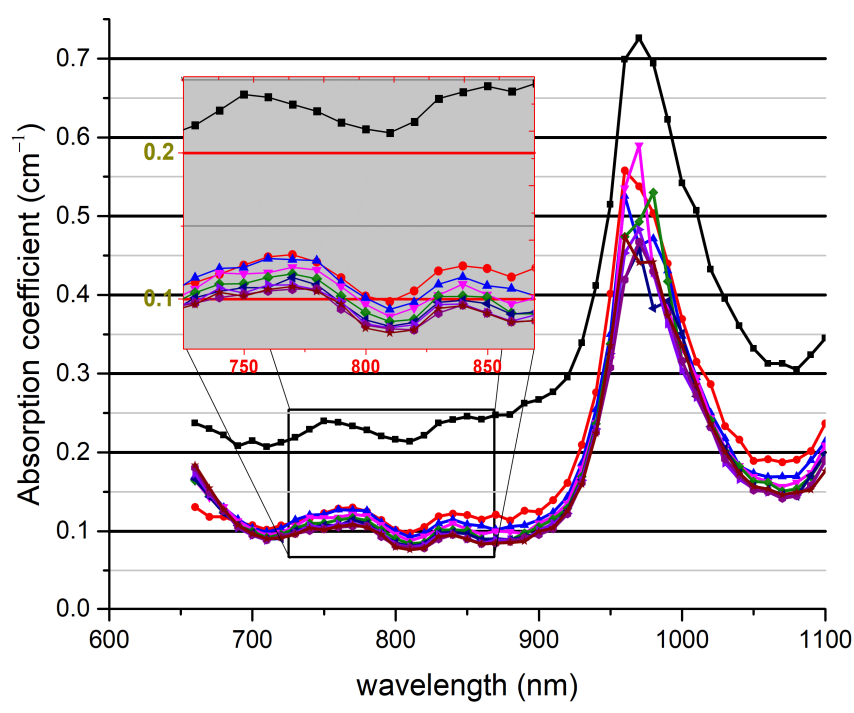

(a)

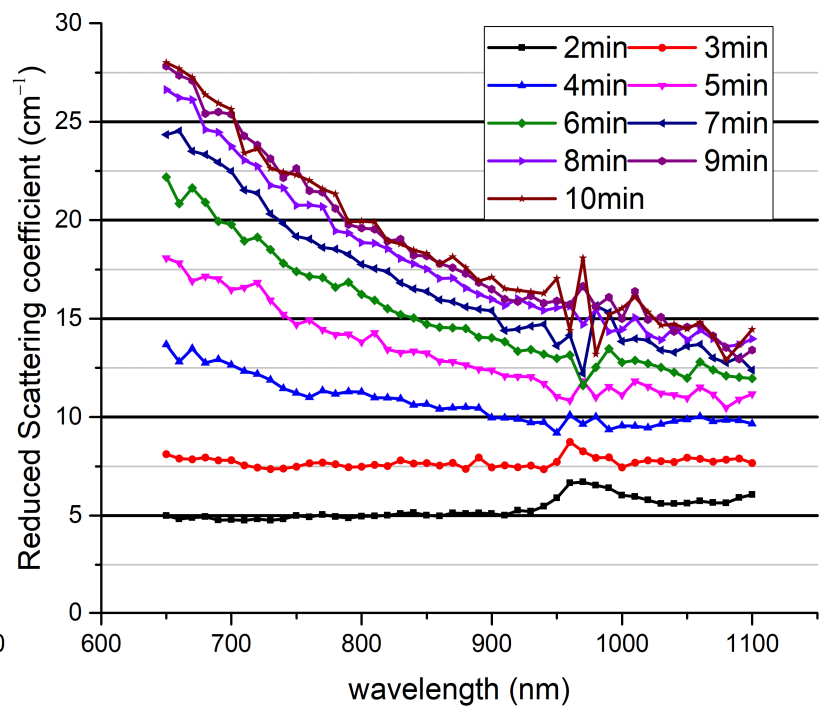

(b)

Figure 2. Broadband (660 to $1100 \mathrm{~nm}$ ) spectral evolution of (a)absorption and (b)reduced scattering coefficients of turkey breast undergoing RFA treatment at a temperature of $70^{\circ} \mathrm{C}$. Both the subplots share the legend at the top-right corner indicating the time elapsed from the begin of the RF treatment.

In conclusion, we have successfully tested the feasibility of using time domain diffuse optical spectroscopy for the monitoring of radiofrequency ablation treatment in biological tissue. While further investigation is necessary, the broadband spectral data seem to provide a number of dependable markers which could help track and improve the efficiency and effectiveness of the RFA treatment in-vivo.

ACKNOWLEDGEMENTS: This work was supported by the European Union's Horizon 2020, Sklodowska-Curie Innovative Training Networks (ITN-ETN) programme, under grant agreement no 675332 BitMap and LaserLab Europe CUSBO002516

\section{REFERENCES}

[1] Curley, S.A., "Radiofrequency Ablation of Malignant Liver Tumors," Annals of Surgical Oncology 10(4), 338347 (2003).

[2] Palumbo, G., et al., "Multidimensional thermal mapping during radiofrequency ablation treatments with minimally invasive fiber optic sensors," Biomedical Optics Express 9(12), 5891 (2018).

[3] Takahashi, H., "Local recurrence after laparoscopic radiofrequency ablation of malignant liver tumors: Results of a contemporary series," Journal of Surgical Oncology 115(7), 830-834 (2017).

[4] Taroni, P., "Breast tissue composition and its dependence on demographic risk factors for breast cancer: noninvasive assessment by time domain diffuse optical spectroscopy.," PloS one 10(6), e0128941 (2015).

[5] Farina, A., et al., "In-vivo multilaboratory investigation of the optical properties of the human head," Biomedical Optics Express 6(7), 2609 (2015).

[6] Konugolu Venkata Sekar, S., et al., "Broadband (600-1350 nm) Time-Resolved Diffuse Optical Spectrometer for Clinical Use,” IEEE Journal on Selected Topics in Quantum Electronics 22(3), (2016). 